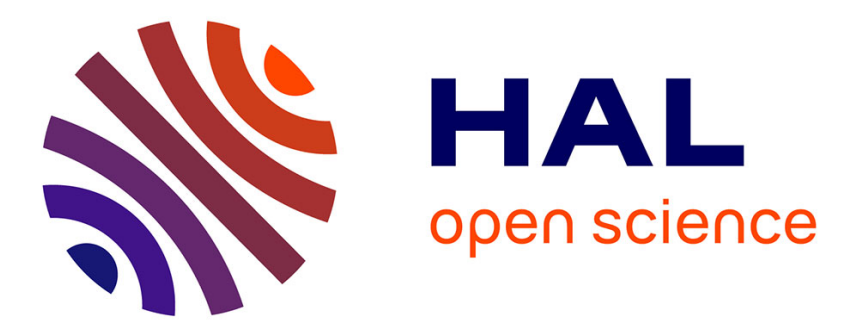

\title{
Dynamical Sparse Signal Recovery with Fixed-Time Convergence
}

Junying Ren, Lei Yu, Chengcheng Lyu, Gang Zheng, Hong Sun, Jean-Pierre Barbot

\section{- To cite this version:}

Junying Ren, Lei Yu, Chengcheng Lyu, Gang Zheng, Hong Sun, et al.. Dynamical Sparse Signal Recovery with Fixed-Time Convergence. Signal Processing, 2019, 162, pp.65-74. 10.1016/j.sigpro.2019.04.010 . hal-02406715

HAL Id: hal-02406715 https://hal.inria.fr/hal-02406715

Submitted on 12 Dec 2019

HAL is a multi-disciplinary open access archive for the deposit and dissemination of scientific research documents, whether they are published or not. The documents may come from teaching and research institutions in France or abroad, or from public or private research centers.
L'archive ouverte pluridisciplinaire HAL, est destinée au dépôt et à la diffusion de documents scientifiques de niveau recherche, publiés ou non, émanant des établissements d'enseignement et de recherche français ou étrangers, des laboratoires publics ou privés. 


\title{
Dynamical Sparse Signal Recovery with Fixed-Time Convergence
}

\author{
Junying Ren ${ }^{\mathrm{a}}$, Lei Yu ${ }^{\mathrm{a}, *}$, Chengcheng Lyu ${ }^{\mathrm{a}}$, Gang Zheng ${ }^{\mathrm{b}}$, Jean-Pierre Barbot ${ }^{\mathrm{b}, \mathrm{c}}$, \\ Hong Sun ${ }^{\mathrm{a}}$ \\ ${ }^{a}$ Signal Processing Laboratory, School of Electronic Information, Wuhan University, Wuhan 430072, China \\ ${ }^{b}$ Non-A team, INRIA-Lille Nord Europe, 40 Avenue Halley, 59650 Villeneuve d'Ascq Cedex, France \\ ${ }^{c}$ Quartz EA 7393, ENSEA, 6 avenue du Ponce du,95014, Cergy Pontoise, France
}

\begin{abstract}
Arising in a large number of application areas, sparse recovery (SR) has been exhaustively investigated and many algorithms have been proposed. Different from the numerical methods realized by iterative algorithm, the recent continuous approach is realized through analog circuit, which takes advantage of short time-delay and fast convergence faster. However, the existing continuous method for SR still has the space to further improve the convergence speed. Consequently, in this paper, we propose a new dynamical continuous system to solve the sparse signal recovery problem with fixed-time convergence property.
\end{abstract}

Keywords: Sparse Recovery, Dynamical System, Fixed-Time Convergence

\section{Introduction}

Over the past few years, the sparse recovery techniques have been thoroughly studied $[\mathbb{I}-\mathbb{Z}]$ and recently employed for several applications, including signal and image processing (e.g., denoising, detection, recognition, and classification) [ㅁ-10]. Consider an unknown $s$-sparse signal $\boldsymbol{\omega}=\left[\omega_{1}, \cdots, \omega_{N}\right]^{T} \in \mathbb{R}^{N}$ which is needed to be recovered,

\footnotetext{
This work is supported by NSFC Grant 61401315, China Scholarship Council and the Project-sponsored by SRF for ROCS, SEM, under Grant 230303.

* Corresponding author

Email addresses: rjy@whu .edu.cn (Junying Ren), ly.wd@whu.edu.cn (Lei Yu), lyu.cc @whu.edu.cn (Chengcheng Lyu), gang.zheng@inria.fr (Gang Zheng), barbot@ensea.fr (Jean-Pierre Barbot), hongsun@whu.edu.cn (Hong Sun)
} 
and suppose that it is acquired from insufficient measurements [II]]

$$
\boldsymbol{y}=\Phi \omega+\varepsilon
$$

where $\boldsymbol{y} \in \mathbb{R}^{M}$ is the observed measurement corrupted by noise $\boldsymbol{\varepsilon} \in \mathbb{R}^{M}$, and $\boldsymbol{\Phi} \in \mathbb{R}^{M \times N}$ is the sensing or measurement matrix. Since $M \ll N$, the recovery of sparse signal $\boldsymbol{\omega}$ from $y$ is an underdetermined problem, the solution of (II) cannot be obtained directly by some linear operations.

The SR problem (II) can be solved by finding the solution of the following unconstrained optimization problem:

$$
\boldsymbol{\omega}^{*}=\arg \min _{\boldsymbol{\omega} \in \mathbb{R}^{N}} \frac{1}{2}\|\boldsymbol{y}-\boldsymbol{\Phi} \boldsymbol{\omega}\|_{2}^{2}+\lambda \rho(\boldsymbol{\omega})
$$

where $\lambda>0$ is a balance parameter and $\rho(\boldsymbol{\omega}): \mathbb{R}^{N} \rightarrow \mathbb{R}_{+}$, is a sparsity-inducing func-

tion, and typically $\rho(\boldsymbol{\omega})=\|\boldsymbol{\omega}\|_{1}=\sum_{i=1}^{N}\left|\omega_{i}\right|$. In order to find the solution of (2), many algorithms have been proposed recently, including the IST[12], BPDN[13], LASSO[14]. However, the aforementioned algorithms are all digital based and normally require a large number of iterations. It may lead very high computational burden and storage requirements, especially encountering applications with huge amount of data, for example, radar imaging [15], FR[116], DOA [177] etc. In the following, we firstly introduce some dynamic systems for SR problem to clarify the motivation.

\subsection{From Digital Algorithm to LCA Dynamical System}

The analog-signal approach, which relies on a continuous dynamical system, is practical due to the instantaneous computation and consequent fast response. For example, the locally competitive algorithm (LCA) algorithm is developed [18-20] to precisely recover the sparse signals with exponential convergence rate.

Let $\dot{f}(\cdot)$ be the derivative with respect to time $t$ of function $f(\cdot)$, the dynamical system proposed in the LCA is given as follows:

$$
\begin{aligned}
& \tau \dot{\boldsymbol{u}}(t)=-\boldsymbol{u}(t)+\left(\boldsymbol{\Phi}^{T} \boldsymbol{\Phi}-I\right) \boldsymbol{a}(t)+\boldsymbol{\Phi}^{T} \boldsymbol{y}, \\
& \hat{\boldsymbol{a}}(t)=\boldsymbol{a}(t)=H_{\lambda}(\boldsymbol{u}(t)),
\end{aligned}
$$

with $\boldsymbol{u} \in \mathbb{R}^{N}$ being the state vector, $\hat{\boldsymbol{a}} \in \mathbb{R}^{N}$ the output of the system, i.e., the estimation of sparse signal $\boldsymbol{\omega}$, and $\tau>0$ a time constant determined by the physical properties of 
the underlying system. We set $\tau=1$ as it does not affect the mathematical analysis of the system. At last, a soft thresholding function is given by

$$
H_{\lambda}(\boldsymbol{u}(t))=\max (|\boldsymbol{u}(t)|-\lambda, 0) \cdot \operatorname{sign}(\boldsymbol{u}(t)) .
$$

where $\lambda>0$ is the threshold parameter, sign is an element-wise operator defined by

$$
\operatorname{sign}(\varphi) \begin{cases}=1 & \text { if } \varphi>0, \\ \in[-1,1] & \text { if } \varphi=0, \\ =-1 & \text { if } \varphi<0 .\end{cases}
$$

Because the equivalence between the equilibrium point of (B]) and the solution of optimization problem (2I), LCA can solve the SR problem [21]. It has been shown that the LCA converges exponentially [2I] to its unique equilibrium point. In control theory, it means that the trajectory of this system will converge to its equilibrium point as $t \rightarrow+\infty$, i.e., $\lim _{t \rightarrow \infty}\|a(t)-\hat{a}(t)\|=0$, which implies a possible infinite convergence time [119]. Consequently, a system for SR with a finite convergence time is sometimes interesting for practical application.

\subsection{From Asymptotic Convergence to Finite-time Convergence}

Compared to LCA that has asymptotic convergence speed, a new dynamical system with faster convergence rate has been proposed in [22], where the proposed system can converge to its equilibrium point in finite time. To clearly present this idea, let us recall firstly the definition of finite-time convergence, which is introduced in control theory.

Definition 1 ([23, 24] $)$. Consider a dynamical system described by $\dot{x}=g(t, x)$ with initial condition $x(0)=x_{0}$, its solution $x\left(t, x_{0}\right)$ is said to be finite-time stable if it is asymptotically stable and reaches equilibrium in a finite time, i.e., $\exists T: \mathbb{R}^{N} \rightarrow \mathbb{R}_{+}$such that $\dot{x}\left(t, x_{0}\right)=0, \forall t \geq T\left(x_{0}\right)$, where $T$ is the settling-time function.

Motivated by the sliding mode technique presented in [22], the differential equation of the dynamical system with finite-time convergence property for SR is constructed as follows:

$$
\begin{aligned}
& \tau \dot{\boldsymbol{u}}(t)=-\left\lceil\boldsymbol{u}(t)+\left(\boldsymbol{\Phi}^{T} \boldsymbol{\Phi}-I\right) \boldsymbol{a}(t)-\boldsymbol{\Phi}^{T} \boldsymbol{y}\right\rfloor^{\alpha}, \\
& \hat{a}(t)=\boldsymbol{a}(t)=H_{\lambda}(\boldsymbol{u}(t)),
\end{aligned}
$$


with the coefficient $\alpha \in \mathbb{R}_{+}, \alpha \in(0,1)$, and $\left\lceil\left.\cdot\right|^{\alpha}\right.$ defined as $\lceil\cdot\rfloor^{\alpha}=|\cdot|^{\alpha} \operatorname{sign}(\cdot)$. When $\alpha=1$, (5) becomes to the LCA system. It has been proved in [22] that the equilibrium point of this finite-time convergence system is also equivalent to the critical points of (2).

The motivation of using sliding mode technique can be presented by two simple systems: $\dot{x}=-\lceil x\rfloor^{\alpha}$ with $\alpha \in(0,1)$, and $\dot{x}=-x$. Although the trajectory of $\dot{x}=-\lceil x\rfloor^{\alpha}$ converges slower to 0 when $|x|>1$, but it will converge to 0 in a finite-time once $|x|<1$, while the trajectory of $\dot{x}=-x$ will maintain the same convergence speed even for $|x|<1$, yielding mathematically a lower convergence when $t \rightarrow \infty$. Despite this, however, the slow convergence speed when $|x|>1$ is still a drawback for $\dot{x}=-\lceil x\rfloor^{\alpha}$. Inspired by that $\dot{x}=-\lceil x\rfloor^{\beta}$ with $\beta \in(1,+\infty)$ is faster when $|x|>1$, thus, adding the other exponential parameters $\beta$ to system (5), we then obtain a new dynamical system with fixed-time convergence property [25].

The key contributions of this paper are summarized as follows: (i) a fixed-time dynamical system with convergence time independent of initial condition is proposed for sparse recovery; (ii) the fixed-time convergence property of the proposed system is proved theoretically.

The rest of this paper is organized as follows. In Section 乙, the proposed system with fixed-time convergence property is described. In Section [3, theoretical results for the proposed system are provided, including some properties of the defined Lyapunov function and the fixed-time convergence of the proposed system. Then in Section 4 , the results in several simulations demonstrate the superiority of our proposed system. Finally, our conclusions are presented in Section [5.

\section{Dynamical System for Sparse Recovery with Fixed-time Convergence}

\subsection{Preliminary of Fixed-time Convergence}

We firstly give the concept of fixed-time stability. It shows that for any initial conditions, dynamical systems with fixed-time stability could converge in a specific time [25]. 
Definition 2 ([25]). For a general dynamical system $\dot{x}=g(t, x), x(0)=x_{0}$, its solution $x\left(t, x_{0}\right)$ is said to be fixed-time stable if it is globally finite-time stable and settling-time function $T\left(x_{0}\right)$ is bounded, i.e., $\exists T_{\max }>0$ such that $T\left(x_{0}\right) \leq T_{\max }, \forall x_{0} \in \mathbb{R}^{N}$.

To investigate the fixed-time convergence problem of any dynamical system, the Lemma $\square$ below could be a determination condition of fixed-time convergence.

Lemma 1 ([26]). Let $V(t)$ be a nonnegative scalar function that is differentiable and satisfies $\dot{V}(t) \leq-\varsigma(V(t))$ with $\varsigma(V)>0, \varsigma(0)=0$; if $t^{*}=\int_{0}^{\infty} \frac{1}{\zeta(V)} d V$ is finite, then for any $V(0)=V_{0}>0, V(t)=0$ for all $t \geq t^{*}$.

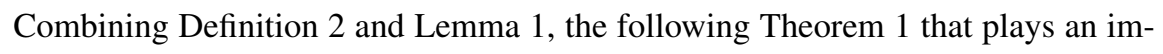
portant role in the proof of fixed-time convergence is obtained. Compared to the theoretical result in [27], the proof here is different and more concise.

Theorem 1. Consider a scalar system

$$
\dot{v} \leq-m v^{\beta}-n v^{\alpha}, v(0)=v_{0} \geq 0,
$$

where $v \geq 0, m>0, n>0, \beta \in[1, \infty]$, and $\alpha \in[0,1]$. The equilibrium of system (16) is globally fixed-time stable with settling time $T$ bounded by

$$
T \leq T_{\max }, T_{\max }=\frac{1}{n(1-\alpha)}+\frac{1}{m(\beta-1)} .
$$

Proof. According to lemma 四, $-m v^{\beta}-n v^{\alpha}=0$ when $v=0$, then we calculate

$$
\begin{aligned}
t^{*} & =\int_{0}^{\infty}\left(m v^{\beta}+n v^{\alpha}\right)^{-1} d v \\
& =\int_{0}^{1}\left(m v^{\beta}+n v^{\alpha}\right)^{-1} d v+\int_{1}^{\infty}\left(m v^{\beta}+n v^{\alpha}\right)^{-1} d v .
\end{aligned}
$$

Combining integral properties, for $v \geq 0$,

$$
\begin{cases}\lim _{k \rightarrow \infty} \int_{1}^{k} v^{-p} d v=\frac{1}{p-1} & p>1, \\ \lim _{k \rightarrow 0} \int_{k}^{1} v^{-p} d v=\frac{1}{1-p} & 0<p<1,\end{cases}
$$

Thus,

$$
\begin{aligned}
& \int_{0}^{1}\left(m v^{\beta}+n v^{\alpha}\right)^{-1} d v+\int_{1}^{\infty}\left(m v^{\beta}+n v^{\alpha}\right)^{-1} d v \\
& \leq \int_{0}^{1}\left(n v^{\alpha}\right)^{-1} d v+\int_{1}^{\infty}\left(m v^{\beta}\right)^{-1} d v \\
& =\frac{1}{n(1-\alpha)}+\frac{1}{m(\beta-1)}
\end{aligned}
$$


Apparently, $t^{*}$ is finite with upper bound $\frac{1}{n(1-\alpha)}+\frac{1}{m(\beta-1)}$, and from Lemma W, $v(t)=0$ for all $t>t^{*}$ and any initial condition $v_{0}$.

\subsection{Dynamical System with Fixed-time Convergence}

In this section, we describe the proposed dynamical system as follows:

$$
\begin{aligned}
& \tau \dot{\boldsymbol{u}}(t)=-\left\lceil\boldsymbol{u}(t)+\left(\boldsymbol{\Phi}^{T} \boldsymbol{\Phi}-I\right) \boldsymbol{a}(t)-\boldsymbol{\Phi}^{T} \boldsymbol{y}\right\rfloor^{\alpha}-\left\lceil\boldsymbol{u}(t)+\left(\boldsymbol{\Phi}^{T} \boldsymbol{\Phi}-I\right) \boldsymbol{a}(t)-\boldsymbol{\Phi}^{T} \boldsymbol{y}\right\rfloor^{\beta} \\
& \hat{a}(t) \boldsymbol{a}(t)=H_{\lambda}(\boldsymbol{u}(t)),
\end{aligned}
$$

where $\alpha \in(0,1], \beta \in[1,+\infty)$, and $\lceil\cdot\rfloor^{\eta}$ with $\eta \in\{\alpha, \beta\}$ is a function defined as

$$
\lceil\cdot\rfloor^{\eta}=|\cdot|^{\eta} \operatorname{sign}(\cdot)
$$

Compared to the finite-time system (5) whose convergence time relies on different initial conditions, the upper bound $T_{\max }$ of (प) can be estimated with any initial condition. In the following sections, we will provide a detailed analysis on the fixed-time convergence property of system (प).

Remark 1. The parameter $\alpha$ guarantees the rapid convergence rate when the system is near to the equilibrium point while the parameter $\beta$ ensures fast convergence when the system is far away from the equilibrium point. Moreover, both $\alpha$ and $\beta$ are essential. If either of the two terms in (Z) is missing or the $\beta$ is too large, the system convergence rate will slow down, and this will be analyzed and demonstrated in next sections.

\section{Fixed-Time Convergence of the Proposed System}

In this section, we firstly prove that the output of system (D) can converge to the critical point of optimization problem (D). Then, the fixed-time convergence of (पD) is presented.

\subsection{Equivalence of Solutions}

Lemma 2. With definition (甘), the equilibrium points of (\) are equal to the critical points of (12). 
Proof. For dynamical system (प्प), any equilibrium point satisfies $\dot{\boldsymbol{u}}(t)=0$, i.e.,

$$
-\boldsymbol{u}+\boldsymbol{a}-\boldsymbol{\Phi}^{T} \boldsymbol{\Phi} \boldsymbol{a}+\boldsymbol{\Phi}^{T} \boldsymbol{y}=0
$$

From (困), $\operatorname{sign}\left(u_{n}\right)=\operatorname{sign}\left(a_{n}\right)$ when $\left|u_{n}\right|>\lambda$ and $u_{n} \in \lambda \operatorname{sign}\left(a_{n}\right)$ when $\left|u_{n}\right| \leq \lambda$. By simple calculate, we can obtain

$$
u_{n}-a_{n}=\left(\left|u_{n}\right|-\max \left(\left|u_{n}\right|-\lambda, 0\right)\right) \operatorname{sign}\left(u_{n}\right)=\lambda \operatorname{sign}\left(a_{n}\right)
$$

Thus, we can say that $\boldsymbol{u}-\boldsymbol{a}$ is the same as $\lambda \operatorname{sign}(\boldsymbol{a})$. Since we have defined $\hat{\boldsymbol{a}}=\boldsymbol{a}=$ $H_{\lambda}(\boldsymbol{u})$ with $\hat{\boldsymbol{a}} \in \mathbb{R}^{N}$ the estimation of sparse signal $\boldsymbol{\omega}$, then the expression (甘) can be represented as

$$
-\lambda \operatorname{sign}(\boldsymbol{\omega})-\boldsymbol{\Phi}^{T} \boldsymbol{\Phi} \boldsymbol{\omega}+\boldsymbol{\Phi}^{T} \boldsymbol{y}=0 .
$$

The sub-differentiation of (ㅁ) with respect to $\boldsymbol{\omega}$ gives

$$
\frac{\partial \frac{1}{2}\|\boldsymbol{y}-\boldsymbol{\Phi} \boldsymbol{\omega}\|_{2}^{2}+\lambda\|\boldsymbol{\omega}\|_{1}}{\partial \boldsymbol{\omega}}=\left(\lambda \operatorname{sign}(\boldsymbol{\omega})+\boldsymbol{\Phi}^{T} \boldsymbol{\Phi} \boldsymbol{\omega}-\boldsymbol{\Phi}^{T} \boldsymbol{y}\right)^{T} .
$$

Consequently, $\frac{\partial \frac{1}{2}\|\boldsymbol{y}-\boldsymbol{\Phi} \boldsymbol{\omega}\|_{2}^{2}+\lambda\|\boldsymbol{\omega}\|_{1}}{\partial \boldsymbol{\omega}}=0$ when $\dot{\boldsymbol{u}}(t)=0$, this ending the proof.

From [19, 28], for most random Gaussian dictionary $\boldsymbol{\Phi}$, the solution of the sparse recovery problem in (2) is unique. On the other hand, Lemma』implies that there exists a unique equilibrium point for (DI) that coincides with the unique critical point of (D2).

\subsection{Convergence Analysis}

In the last subsection, it has been shown that there exists a unique equilibrium point $\boldsymbol{u}^{*}$ of dynamical system (प). Using $\boldsymbol{u}^{*}$ and its corresponding $\boldsymbol{a}^{*}$, the error terms and a function $E(\tilde{\boldsymbol{u}})$ are introduced to analyze the fixed-time convergence property of system (पD):

$$
\begin{gathered}
\tilde{\boldsymbol{u}}(t):=\boldsymbol{u}(t)-\boldsymbol{u}^{*}, \\
\tilde{\boldsymbol{a}}(t):=\boldsymbol{a}(t)-\boldsymbol{a}^{*}, \\
E(\tilde{\boldsymbol{u}}(t)):=\frac{1}{2}\|\tilde{\boldsymbol{u}}(t)\|_{2}^{2}+\operatorname{Tr}\left\{\left(\boldsymbol{\Phi}^{T} \boldsymbol{\Phi}-I\right) \boldsymbol{G}(\tilde{\boldsymbol{u}}(t))\right\}
\end{gathered}
$$


where the matrix $G(\tilde{u})$ is defined as

$$
\boldsymbol{G}(\tilde{\boldsymbol{u}}(\boldsymbol{t}))=\left[\begin{array}{cccc}
G_{1}\left(\tilde{u}_{1}(t)\right) & G_{1}\left(\tilde{u}_{2}(t)\right) & \ldots & G_{1}\left(\tilde{u}_{N}(t)\right) \\
G_{2}\left(\tilde{u}_{1}(t)\right) & G_{2}\left(\tilde{u}_{2}(t)\right) & \ldots & G_{2}\left(\tilde{u}_{N}(t)\right) \\
\vdots & \vdots & \ddots & \vdots \\
G_{N}\left(\tilde{u}_{1}(t)\right) & G_{N}\left(\tilde{u}_{2}(t)\right) & \ldots & G_{N}\left(\tilde{u}_{N}(t)\right)
\end{array}\right]^{T}
$$

with

$$
G_{i}\left(\tilde{u}_{j}(t)\right)= \begin{cases}\int_{0}^{\tilde{u}_{i}(t)} g_{i}(s) d s, & i=j \\ \int_{\bar{u}_{j}}^{\tilde{u}_{j}(t)} g_{i}\left(\rho_{i j}(s)\right) d s, & i \neq j\end{cases}
$$

where $g_{i}(s)=H_{\lambda}\left(s+u_{i}^{*}\right)-H_{\lambda}\left(u_{i}^{*}\right), \bar{u}_{j}$ is the $j$-th component of some constant vector $\overline{\boldsymbol{u}}$, and $\rho_{i j}$ is the function related to the trajectory $\tilde{\boldsymbol{u}}(t)$ such that $\tilde{u}_{i}(t)=\rho_{i j}\left(\tilde{u}_{j}(t)\right)$.

Moreover, denote $\delta$ the smallest positive constant such that for any active set $\Gamma(t)$ visited by the algorithm and any vector $\boldsymbol{x}$ in $\mathbb{R}^{N}$, we have

$$
(1-\delta)\|\boldsymbol{x}\|_{2}^{2} \leq\|\boldsymbol{\Phi} \boldsymbol{x}\|_{2}^{2} \leq(1+\delta)\|\boldsymbol{x}\|_{2}^{2}
$$

so the $\delta$ depends on the singular values of the matrix $\boldsymbol{\Phi}_{\Gamma(t)}$. Then we have the following lemma:

Lemma 3. The proposed dynamical system in (प) and the function $E$ in (पD) satisfies the following properties:

1) For all $\tilde{u}_{i}, 0 \leq G_{i}\left(\tilde{u}_{i}(t)\right) \leq \frac{\tilde{u}_{i}^{2}(t)}{2}$,

2)Under definition ( [2), then $\left\|\tilde{\boldsymbol{u}}+\left(\boldsymbol{\Phi}^{T} \boldsymbol{\Phi}-I\right) \tilde{\boldsymbol{a}}\right\|_{2}^{2} \geq(1-\boldsymbol{\delta})\|\tilde{\boldsymbol{u}}\|_{2}^{2}$,

3) There exists a positive constant $\gamma>0$ such that $E(\tilde{\boldsymbol{u}}) \leq \gamma\|\tilde{\boldsymbol{u}}\|_{2}^{2}$,

4) For dynamical system (प), $\dot{E} \leq 0$, and $E$ cannot be negative, i.e. $E \geq 0$,

Proof. 1) According to (四), we have

$$
H_{\lambda}(x)-H_{\lambda}(y)= \begin{cases}x-y-\lambda \operatorname{sign}(x)+\lambda \operatorname{sign}(y), & |x|>\lambda,|y|>\lambda \\ x-\lambda \operatorname{sign}(x), & |x|>\lambda,|y| \leq \lambda \\ -y+\lambda \operatorname{sign}(y), & |x| \leq \lambda,|y|>\lambda \\ 0, & |x| \leq \lambda,|y| \leq \lambda\end{cases}
$$


which implies that $H_{\lambda}(x)-H_{\lambda}(y) \leq x-y$ for $\forall x \geq y$, furthermore,

$$
\left|\tilde{a}_{i}(t)\right|=\left|g_{i}\left(\tilde{u}_{i}(t)\right)\right| \leq\left|\tilde{u}_{i}(t)\right|, \forall \tilde{u}_{i}(t)
$$

And as the operator $H_{\lambda}\left(u_{i}\right)$ is non-decreasing with respect to $u_{i}$, it is obvious that $\tilde{u}_{i}(t) \cdot g_{i}\left(\tilde{u}_{i}(t)\right) \geq 0$, thus we have two cases:

- when $\tilde{u}_{i}(t) \leq 0$, then $g_{i}\left(\tilde{u}_{i}(t)\right) \leq 0$ and $g_{i}\left(\tilde{u}_{i}(t)\right) \geq \tilde{u}_{i}(t)$,

$$
G_{i}\left(\tilde{u}_{i}(t)\right)=\int_{0}^{\tilde{u}_{i}(t)} g_{i}(s) d s=\int_{\tilde{u}_{i}(t)}^{0}\left(-g_{i}(s)\right) d s \Rightarrow 0 \leq G_{i}\left(\tilde{u}_{i}(t)\right) \leq \frac{\tilde{u}_{i}(t)^{2}}{2}
$$

- when $\tilde{u}_{i}(t) \geq 0$, then $g_{i}\left(\tilde{u}_{i}(t)\right) \geq 0$ and $g_{i}\left(\tilde{u}_{i}(t)\right) \leq \tilde{u}_{i}(t)$,

$$
G_{i}\left(\tilde{u}_{i}(t)\right)=\int_{0}^{\tilde{u}_{i}(t)} g_{i}(s) d s \Rightarrow 0 \leq G_{i}\left(\tilde{u}_{i}(t)\right) \leq \frac{\tilde{u}_{i}(t)^{2}}{2}
$$

2) For $\forall \tilde{\boldsymbol{u}}$, we have

$$
\left\|\tilde{\boldsymbol{u}}+\left(\boldsymbol{\Phi}^{T} \boldsymbol{\Phi}-I\right) \tilde{\boldsymbol{a}}\right\|_{2}^{2} \geq\|\tilde{\boldsymbol{u}}\|_{2}^{2}-\left\|\left(\boldsymbol{\Phi}^{T} \boldsymbol{\Phi}-I\right) \tilde{\boldsymbol{a}}\right\|_{2}^{2}
$$

From (12), then

$$
\left\|\left(\boldsymbol{\Phi}^{T} \boldsymbol{\Phi}-I\right) \tilde{\boldsymbol{a}}\right\|_{2}^{2} \leq \delta\|\tilde{\boldsymbol{a}}\|_{2}^{2}
$$

Considering the worst case of the inclusion, we will get

$$
\left\|\tilde{\boldsymbol{u}}+\left(\boldsymbol{\Phi}^{T} \boldsymbol{\Phi}-I\right) \tilde{\boldsymbol{a}}\right\|_{2}^{2} \geq\|\tilde{\boldsymbol{u}}\|_{2}^{2}-\delta\|\tilde{\boldsymbol{a}}\|_{2}^{2}
$$

Moreover, by ([13),

$$
\|\tilde{\boldsymbol{a}}\|_{2}^{2} \leq\|\tilde{\boldsymbol{u}}\|_{2}^{2}
$$

then

$$
\left\|\tilde{\boldsymbol{u}}+\left(\boldsymbol{\Phi}^{T} \boldsymbol{\Phi}-I\right) \tilde{\boldsymbol{a}}\right\|_{2}^{2} \geq(1-\delta)\|\tilde{\boldsymbol{u}}\|_{2}^{2}
$$

3)As the same to Lemma 3 in [22], the system (प1) is bounded, thus $\tilde{\boldsymbol{a}}(t)$ and $\tilde{\boldsymbol{u}}(t)$ is always bounded, i.e., for $\forall \sigma, \exists \kappa_{\tilde{u}(t)}, \kappa_{\tilde{u}(t)}^{\prime}>0$ such that $\left|\tilde{a}_{i}(\sigma)\right| \leq \kappa_{\tilde{u}(t)}|| \tilde{u}(t) \|_{2}$ and $\left|\tilde{u}_{j}(t)-\bar{u}_{j}\right| \leq \kappa_{\tilde{u}(t)}^{\prime}|| \tilde{u}(t) \|_{2}$. Thus,

$$
\begin{aligned}
\left|G_{i}\left(\tilde{u}_{j}\right)\right| & =\left|\int_{\bar{u}_{j}}^{\tilde{u}_{j}(t)} g_{i}\left(\rho_{i j}(s)\right) d s\right| \leq \int_{\bar{u}_{j}}^{\tilde{u}_{j}(t)}\left|g_{i}\left(\rho_{i j}(s)\right)\right| d s \\
& \leq \kappa_{\tilde{u}(t)}|| \tilde{u}(t)||_{2}\left|\int_{\bar{u}_{j}}^{\tilde{u}_{j}(t)} d s\right|=\kappa_{\tilde{u}(t)}|| \tilde{u}(t)||_{2}\left|\tilde{u}_{j}(t)-\bar{u}_{j}\right| \\
& \leq \kappa_{\tilde{u}(t)} \kappa_{\tilde{u}(t)}^{\prime}|| \tilde{u}(t) \|_{2}^{2}
\end{aligned}
$$


Combine property 1 , let $\kappa=\max \left\{\kappa_{\tilde{u}(t)} \kappa_{\tilde{u}(t)}^{\prime}, 1 / 2\right\}$, then

$$
\left|G_{i}\left(\tilde{u}_{j}(t)\right)\right| \leq \kappa \tilde{u}_{i}^{2}(t), \forall i, j
$$

Exploiting the coherence property of matrix $\Phi^{\square}$, we can get

$$
\begin{aligned}
\left|\operatorname{Tr}\left(\boldsymbol{\Phi}^{T} \boldsymbol{\Phi} G\right)\right| & =\left|\sum_{i=1}^{N} \sum_{j=1}^{N} \phi_{i}^{T} \phi_{j} G_{i}\left(\tilde{u}_{j}(t)\right)\right| \\
& \leq \sum_{i=1}^{N} \sum_{j=1}^{N}\left|\phi_{i}^{T} \phi_{j}\right| \cdot\left|G_{i}\left(\tilde{u}_{j}(t)\right)\right| \\
& \leq \mu \kappa N\|\tilde{\boldsymbol{u}}(t)\|_{2}^{2}
\end{aligned}
$$

Using property 1 , we can easily conclude that

$$
\operatorname{Tr}(\boldsymbol{G})=\sum_{i=1}^{N} G_{i}\left(\tilde{u}_{i}(t)\right) \geq 0
$$

With the results of (ㅁ) and (피), we can obtain that

$$
\begin{aligned}
|E(\tilde{\boldsymbol{u}}(t))| & \leq \frac{1}{2}\|\tilde{\boldsymbol{u}}(t)\|_{2}^{2}+\operatorname{Tr}\left(\boldsymbol{\Phi}^{T} \boldsymbol{\Phi} G\right) \\
& \leq \frac{1}{2}\|\tilde{\boldsymbol{u}}(t)\|_{2}^{2}+\mu \kappa N\|\tilde{\boldsymbol{u}}(t)\|_{2}^{2} \\
& =\frac{1+2 \mu \kappa N}{2}\|\tilde{\boldsymbol{u}}(t)\|_{2}^{2}
\end{aligned}
$$

Consequently, there exists a positive constant $\gamma=\frac{1+2 \mu \kappa N}{2}$ such that $E(\tilde{\boldsymbol{u}}(t)) \leq \gamma\|\tilde{\boldsymbol{u}}\|_{2}^{2}$.

4) Firstly, according to the derivative rule of the variable limit integral,

$$
\begin{aligned}
\frac{d G_{i}\left(\tilde{u}_{j}(t)\right)}{d t} & =\frac{d G_{i}\left(\tilde{u}_{j}(t)\right)}{d \tilde{u}_{j}} \frac{d \tilde{u}_{j}}{d t} \\
& =\frac{d \int_{c}^{\tilde{u}_{j}(t)} g_{i}\left(\rho_{i j}(s)\right) d s}{d \tilde{u}_{j}} \dot{\tilde{u}}_{j} \\
& =g_{i}\left(\rho_{i j}\left(\tilde{u}_{j}\right)\right) \dot{\tilde{u}}_{j}=\tilde{a}_{i} \dot{\tilde{u}}_{j}
\end{aligned}
$$

\footnotetext{
${ }^{1}$ If $\boldsymbol{\Phi}$ satisfies $R I P$ with constant $\delta$, then the coherence of $\boldsymbol{\Phi}$ defined as $\mu=\max _{i \neq j} \frac{\left|\left\langle\phi_{i}, \phi_{j}\right\rangle\right|}{\left\|\phi_{i}\right\|_{2}\left\|\phi_{j}\right\|_{2}}$ has the value of $\mu=\delta /(s-1)$, and if columns of $\boldsymbol{\Phi}$ are not normalized, then $\left|\left\langle\phi_{i}, \phi_{j}\right\rangle\right| \leq \mu \rho^{2}$ with $\rho=\max \left\|\phi_{i}\right\|_{2}$.
} 
Consequently,

$$
\begin{aligned}
\frac{d \operatorname{Tr}\left\{\left(\boldsymbol{\Phi}^{T} \boldsymbol{\Phi}-I\right) \boldsymbol{G}(\tilde{\boldsymbol{u}})\right\}}{d t} & =\frac{d \sum_{i=1}^{N} \sum_{j=1}^{N} \phi_{i}^{T} \phi_{j} G_{i}\left(\tilde{u}_{j}(t)\right)}{d t}-\frac{d \sum_{i=1}^{N} G_{i}\left(\tilde{u}_{i}(t)\right)}{d t} \\
& =\sum_{i=1}^{N} \sum_{j=1}^{N} \phi_{i}^{T} \phi_{j} \tilde{a}_{i} \dot{\tilde{u}}_{j}-\sum_{i=1}^{N} \tilde{a}_{i} \dot{\tilde{u}}_{i} \\
& =\left(\left(\boldsymbol{\Phi}^{T} \boldsymbol{\Phi}-I\right) \tilde{\boldsymbol{a}}\right)^{T} \dot{\tilde{\boldsymbol{u}}}(t)
\end{aligned}
$$

Then from (III), the time derivative of $E$ results in

$$
\dot{E}=\frac{d E^{T}}{d \tilde{\boldsymbol{u}}} \frac{d \tilde{\boldsymbol{u}}}{d t}=\left(\tilde{\boldsymbol{u}}+\left(\boldsymbol{\Phi}^{T} \boldsymbol{\Phi}-I\right) \tilde{\boldsymbol{a}}\right)^{T} \dot{\tilde{\boldsymbol{u}}}(t) .
$$

Given that $\boldsymbol{u}^{*}$ is constant we have

$$
\dot{\tilde{\boldsymbol{u}}}=\dot{\boldsymbol{u}}=-\left\lceil\boldsymbol{u}(t)+\left(\boldsymbol{\Phi}^{T} \boldsymbol{\Phi}-I\right) \boldsymbol{a}(t)-\boldsymbol{\Phi}^{T} \boldsymbol{y}\right\rfloor^{\alpha}-\left\lceil\boldsymbol{u}(t)+\left(\boldsymbol{\Phi}^{T} \boldsymbol{\Phi}-I\right) \boldsymbol{a}(t)-\boldsymbol{\Phi}^{T} \boldsymbol{y}\right\rfloor^{\beta} .
$$

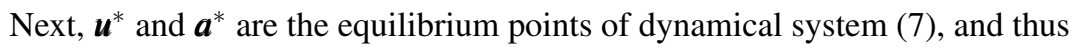

$$
\boldsymbol{u}^{*}+\left(\boldsymbol{\Phi}^{T} \boldsymbol{\Phi}-I\right) \boldsymbol{a}^{*}-\boldsymbol{\Phi}^{T} \boldsymbol{y}=0 .
$$

Substituting (21) in (201), we obtain

$$
\dot{\tilde{\boldsymbol{u}}}=-\left\lceil\tilde{\boldsymbol{u}}+\left(\boldsymbol{\Phi}^{T} \boldsymbol{\Phi}-I\right) \tilde{\boldsymbol{a}}\right\rfloor^{\alpha}-\left\lceil\tilde{\boldsymbol{u}}+\left(\boldsymbol{\Phi}^{T} \boldsymbol{\Phi}-I\right) \tilde{\boldsymbol{a}}\right\rfloor^{\beta} .
$$

Consequently,

$$
\begin{aligned}
\dot{E} & \left.\left.=-\left(\tilde{\boldsymbol{u}}+\left(\boldsymbol{\Phi}^{T} \boldsymbol{\Phi}-I\right) \tilde{\boldsymbol{a}}\right)^{T}\left[\tilde{\boldsymbol{u}}+\left(\boldsymbol{\Phi}^{T} \boldsymbol{\Phi}-I\right) \tilde{\boldsymbol{a}}\right]^{\alpha}+\mid \tilde{\boldsymbol{u}}+\left(\boldsymbol{\Phi}^{T} \boldsymbol{\Phi}-I\right) \tilde{\boldsymbol{a}}\right]^{\beta}\right] \\
& =-\left|\tilde{\boldsymbol{u}}+\left(\boldsymbol{\Phi}^{T} \boldsymbol{\Phi}-I\right) \tilde{\boldsymbol{a}}\right|^{T}\left[\left|\tilde{\boldsymbol{u}}+\left(\boldsymbol{\Phi}^{T} \boldsymbol{\Phi}-I\right) \tilde{\boldsymbol{a}}\right|^{\alpha}+\left|\tilde{\boldsymbol{u}}+\left(\boldsymbol{\Phi}^{T} \boldsymbol{\Phi}-I\right) \tilde{\boldsymbol{a}}\right|^{\beta}\right] .
\end{aligned}
$$

Consequently, it's obviously that $\dot{E} \leq 0$.

Moreover, as the same result of Lemma 3 in [22], the proposed system (पd) is Lyapunov stable for any initial condition. And we also proved the boundness of $E$ in the previous property. Consequently, according to the Lasalle theorem [29], system (DI) will converge to some invariant set $M$,

$$
M=\left\{\tilde{\boldsymbol{u}} \mid \dot{E}(\tilde{\boldsymbol{u}})=\tilde{\boldsymbol{u}}+\left(\boldsymbol{\Phi}^{T} \boldsymbol{\Phi}-I\right) \tilde{\boldsymbol{a}}=0\right\}
$$

Particularly, since the lemma $\rrbracket$ implies that system (पI) has only one unique solution, thus the equilibrium point for $E$ is unique, i.e. the invariant set $M$ has only one unique element, which is $\tilde{\boldsymbol{u}}=0$ where $E(0)=0$. 
Furthermore, we know that $\dot{E} \leq 0$ for all $\tilde{\boldsymbol{u}}$. Consequently, for any $E<0$, it will be non-increasing all the time instead of converging to 0 , i.e., the system will not converge, which is contradictory. Thus for the proposed dynamic system $(\mathbb{Z}), E$ is non-negative, i.e. $E \geq 0$.

With the previous results, now we can prove the fixed-time convergence of the proposed system (प).

Theorem 2. For dynamical system (प) with definition (甘), for any initial condition $u_{0}$, there is a bound time $T$, where $T \leq T_{\max }$ with $T_{\max }=\frac{2 \gamma}{1-\delta}\left(\frac{1}{1-\alpha}+\frac{N^{(\beta-1) / 2}}{\beta-1}\right)$, when $t \geq T$, one has $E(\tilde{\boldsymbol{u}}(t))=0$.

Proof. From ([23),

$$
\begin{aligned}
\dot{E}(\tilde{u}(t)) & =-\left(\tilde{\boldsymbol{u}}+\left(\boldsymbol{\Phi}^{T} \boldsymbol{\Phi}-I\right) \tilde{\boldsymbol{a}}\right)^{T}\left[\left[\tilde{\boldsymbol{u}}+\left(\boldsymbol{\Phi}^{T} \boldsymbol{\Phi}-I\right) \tilde{\boldsymbol{a}}\right]^{\alpha}+\left\lceil\tilde{\boldsymbol{u}}+\left(\boldsymbol{\Phi}^{T} \boldsymbol{\Phi}-I\right) \tilde{\boldsymbol{a}}\right]^{\beta}\right] \\
& =-\left\|\tilde{\boldsymbol{u}}+\left(\boldsymbol{\Phi}^{T} \boldsymbol{\Phi}-I\right) \tilde{\boldsymbol{a}}\right\|_{1+\alpha}^{1+\alpha}-\left\|\tilde{\boldsymbol{u}}+\left(\boldsymbol{\Phi}^{T} \boldsymbol{\Phi}-I\right) \tilde{\boldsymbol{a}}\right\|_{1+\beta}^{1+\beta} .
\end{aligned}
$$

According to Lemma 3.3 and 3.4 in [30], for $\forall \xi_{i} \geq 0, i=1, \cdots, N$,

$$
\begin{cases}\sum_{i=1}^{N} \xi_{i}^{p} \geq\left(\sum_{i=1}^{N} \xi_{i}\right)^{p} & 0<p \leq 1 \\ \sum_{i=1}^{N} \xi_{i}^{p} \geq N^{1-p}\left(\sum_{i=1}^{N} \xi_{i}\right)^{p} & p \geq 1\end{cases}
$$

Note that $0<(1+\alpha) / 2 \leq 1<(1+\beta) / 2$, define $\tilde{\boldsymbol{u}}+\left(\boldsymbol{\Phi}^{T} \boldsymbol{\Phi}-I\right) \tilde{\boldsymbol{a}}$ as $\boldsymbol{B}$, then we have

$$
\left\{\begin{array}{l}
\sum_{i=1}^{N}\left(B_{i}^{2}\right)^{(1+\alpha) / 2} \geq\left(\sum_{i=1}^{N} B_{i}^{2}\right)^{(1+\alpha) / 2} \\
\sum_{i=1}^{N}\left(B_{i}^{2}\right)^{(1+\beta) / 2} \geq N^{(1-\beta) / 2}\left(\sum_{i=1}^{N} B_{i}^{2}\right)^{(1+\beta) / 2}
\end{array}\right.
$$

which means that

$$
\begin{aligned}
& \left\|\tilde{\boldsymbol{u}}+\left(\boldsymbol{\Phi}^{T} \boldsymbol{\Phi}-I\right) \tilde{\boldsymbol{a}}\right\|_{1+\alpha}^{1+\alpha} \geq\left\|\tilde{\boldsymbol{u}}+\left(\boldsymbol{\Phi}^{T} \boldsymbol{\Phi}-I\right) \tilde{\boldsymbol{a}}\right\|_{2}^{1+\alpha} \\
& \left\|\tilde{\boldsymbol{u}}+\left(\boldsymbol{\Phi}^{T} \boldsymbol{\Phi}-I\right) \tilde{\boldsymbol{a}}\right\|_{1+\beta}^{1+\beta} \geq N^{(1-\beta) / 2}\left\|\tilde{\boldsymbol{u}}+\left(\boldsymbol{\Phi}^{T} \boldsymbol{\Phi}-I\right) \tilde{\boldsymbol{a}}\right\|_{2}^{1+\beta}
\end{aligned}
$$

From the property 2) and 3) of lemma B, $\left\|\tilde{\boldsymbol{u}}+\left(\boldsymbol{\Phi}^{T} \boldsymbol{\Phi}-I\right) \tilde{\boldsymbol{a}}\right\|_{2}^{2} \geq \frac{1-\delta}{\gamma} E(\tilde{\boldsymbol{u}}(t))$, then ([24) becomes

$$
\dot{E}(\tilde{\boldsymbol{u}}(t)) \leq-\frac{1-\delta}{\gamma}\left(E(\tilde{\boldsymbol{u}}(t))^{(1+\alpha) / 2}+N^{(1-\beta) / 2} E(\tilde{\boldsymbol{u}}(t))^{(1+\beta) / 2}\right) .
$$


From the property 4) of lemma [, $E \geq 0$. Then, according to Theorem [, there is a bound time $T$, such that

$$
T \leq T_{\max }=\frac{2 \gamma}{1-\delta}\left(\frac{1}{1-\alpha}+\frac{N^{(\beta-1) / 2}}{\beta-1}\right) .
$$

Finally, according to Lemma आ, $E=0$ for all $t \geq T$ and any initial condition. This completes the proof.

Remark 2. From (26), it can be found that the $T_{\max }$ is independent of the initial conditions. In addition, the $T_{\max }$ is smaller with smaller $\alpha$. Furthermore, calculating the derivative of $T_{\max }$ about $\beta$, we get

$$
\frac{d T_{\max }}{d \beta}=\imath \frac{N^{(\beta-1) / 2}}{\beta-1}\left(\frac{\ln N}{2}-\frac{1}{\beta-1}\right)
$$

with $\imath=\frac{2 \gamma}{1-\delta}>0$. This means that when $\beta=1+\frac{2}{\ln N}$, the $T_{\max }$ is minimum. Furthermore, if $N>e^{2}$, with e the irrational number $2.71828 \ldots$, then $1+\frac{2}{\ln N} \in(1,2)$, and the larger $N$, the smaller $1+\frac{2}{\ln N}$. Later in this paper, we will further verify these conclusions through experiments.

\section{Simulations and Results}

In this section, the superior performance of the proposed fixed-time convergence system is demonstrated through several simulations.

The experiments will be executed according to following settings. Sparse signals $\boldsymbol{a} \in \mathbb{R}^{N}$ with length $N=200$ and sparsity $s=10$ are randomly generated, whose nonzero entries are drawn from a normal Gaussian distribution. Measurements $\boldsymbol{y} \in \mathbb{R}^{M}$ with $M=100$ are collected via random projection $\boldsymbol{y}=\boldsymbol{\Phi} \boldsymbol{a}+\boldsymbol{\varepsilon}$, where the measurement matrix $\boldsymbol{\Phi} \in \mathbb{R}^{M \times N}$, columns of which are normalized, is generated from a normal Gaussian distribution and $\varepsilon$ is the Gaussian noise with standard derivation $\sigma=0.016$. The LCA, the finite-time convergence system, and the proposed fixed-time convergence system are simulated through a discrete approximation of ODE45 solver in MATLAB with fixed sampling time equal to 0.1 . The threshold value is set as $\lambda=0.05$ and the initial condition $\boldsymbol{u}(0)$ is generated randomly if no specific statement is made. Especially for section 4.1 and $4.2, \boldsymbol{u}(0)=\mathbf{0}$. 
The experimental results can be divided into four parts: (i) the SR performance of the proposed system is compared with LCA; (ii) the convergence rate with different parameters is analyzed, e.g., number of observations $M$, the signal length $N$, and the sparsity level $s$; (iii) the fixed-time convergence property of the proposed system is presented; (iv) the influence of $\alpha$ and $\beta$ on convergence property is illustrated.

\subsection{Sparse Recovery}

As stated in Lemma \, the proposed dynamical system can converge to the solution of a general SR optimization problem. In this simulation, we examined the recovery performance of the LCA and the proposed system (पd) under the same initial condition. With comparison to the original sparse signal, the results presented in Fig. 1 show that the proposed system can reconstruct the sparse signal as effectively as the LCA.

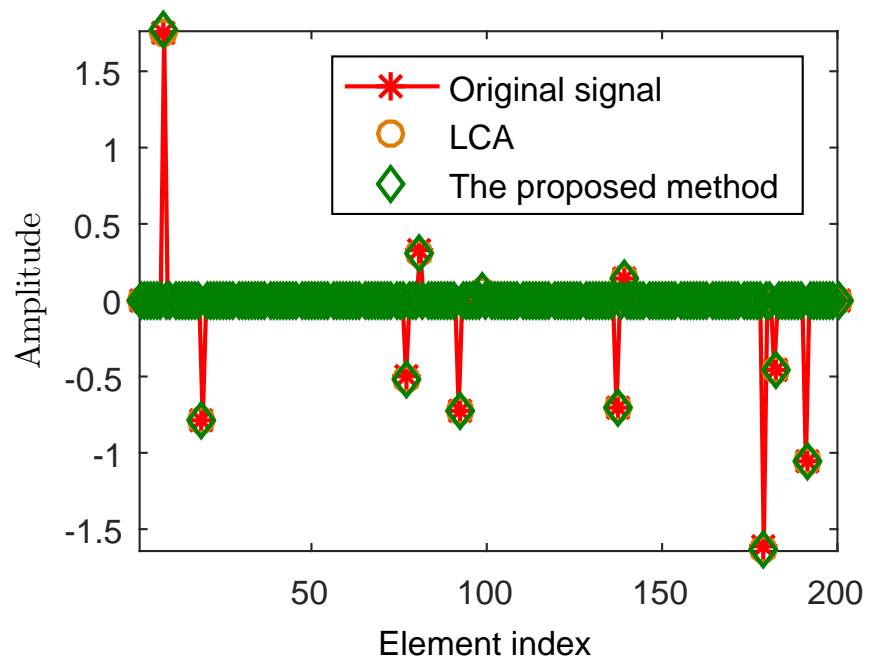

Figure 1: The sparse signal $a^{*}$ retrieved by the proposed fixed-time system and the LCA.

\subsection{Convergence Rate with Different Parameters}

In this section, we'll obtain the convergence rate with different values of $M, N$, and $s$. The error in this simulation was calculated as $\log _{10}\left\|\boldsymbol{u}-\boldsymbol{u}^{*}\right\|_{2}^{2}$. Set $\alpha=0.2$ and $\beta=3$, then vary one of $M, N$, and $s$ while the other two are set to the default. Set $M$ to 
$[90,130,160,175], N$ to $[200,250,300,350]$, and $s$ to $[5,10,15,20]$, then the simulation results are shown in Fig. \. which reflected that the convergence rate is getting faster with the increase of $M$ or the decrease of $N$ and $s$. It is in accordance with the fact that the more information we have or the less information we need to recover, the easier and faster the process will be to recover the sparse signal.

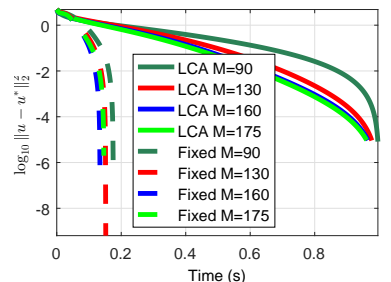

(a)

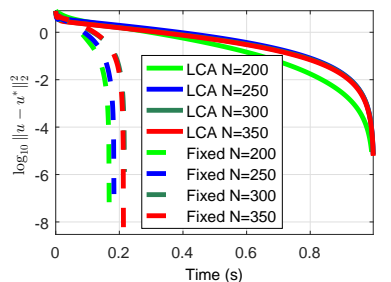

(b)

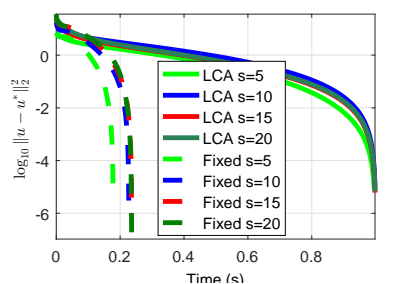

(c)

Figure 2: Convergence of $\left\|u-u^{*}\right\|_{2}^{2}$ for the proposed dynamical system (dashed lines) and the LCA (solid lines) with $\alpha=0.2$ and $\beta=5$ when simulation settings vary with respect to (a) the number of observations $M$, (b) the signal length $N$, and (c) the sparsity level $s$, respectively.

\subsection{Fixed-Time Convergence}

In this section, to expound the fixed-time convergence of the proposed system, we'll firstly demonstrate that the proposed system is the fastest one with respect to the same parametric environment. Set $\beta=3, \alpha=0.2$, and the initial condition $\boldsymbol{u}(0)$ is drawn from a Gaussian distribution with standard derivation $\sigma=3$. Fig. [ indeed reflects that the system with fixed-time convergence property is faster than the other two systems, whether when $\boldsymbol{u}(t)$ is far from or near (the zoom window) to the equilibrium point since the $\alpha$ and $\beta$ work together.

In order to exhibit what happens during the process of convergence and more particularly to understand how the algorithm works with respect to the active node set, the error between $\hat{\boldsymbol{a}}(t)$ and $\boldsymbol{a}^{*}$ is calculated by $\sum_{i=1}^{N}\left(\left|\hat{\boldsymbol{a}}_{i}\right|_{0}-\left|\boldsymbol{a}_{i}^{*}\right|_{0}\right)$, with $\left|\hat{\boldsymbol{a}}_{i}\right|_{0}=0$ if $\hat{\boldsymbol{a}}_{i}=0$, otherwise $\left|\hat{\boldsymbol{a}}_{i}\right|_{0}=1$. Set $\beta=2, \alpha=0.5$, and all nodes of the estimation are initialized to 1 , then Fig. Thb shows that the error of the $\ell_{0}$ norm between $a$ and $\hat{a}$ progressively decreases to zero. Moreover, the convergence process of $\log _{10}\|\boldsymbol{u}(t)-\boldsymbol{u}(t-1)\|_{2}^{2}$ in

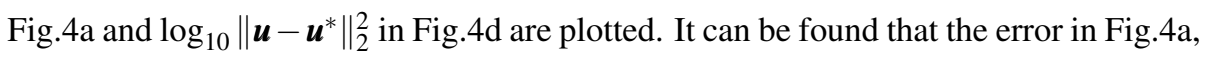




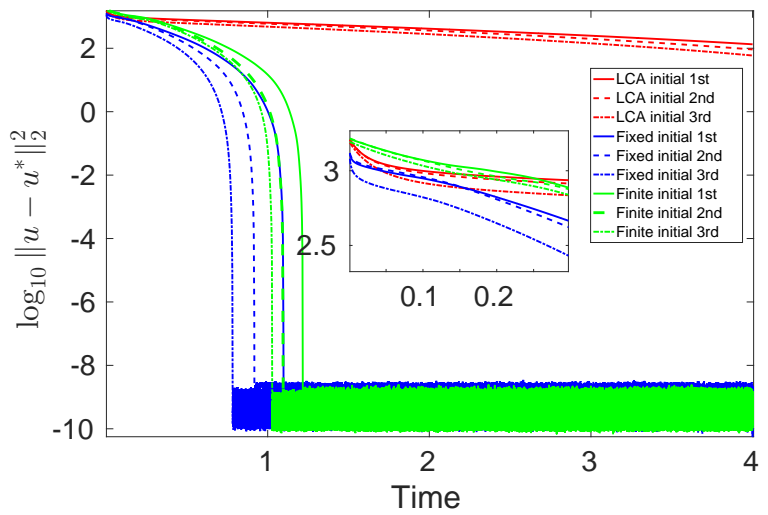

Figure 3: Convergence of $\left\|u-u^{*}\right\|_{2}^{2}$ for the proposed dynamical system under different initial conditions comparing with other methods.

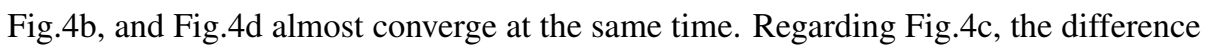
between the original signal and the fixed-time estimation is due to the threshold $\lambda$, and it is natural to compensate this error as follows: design a new estimation $\tilde{a}$, which can be calculated as: $\tilde{a}=\hat{a}+\lambda \operatorname{sign}(\hat{a})$ if $|\hat{a}|>\lambda$, otherwise $\tilde{a}=\hat{a}$.

As the same to Fig.[1 except initializing all node of the estimation to 0, Fig. [1] shows that although the initial value of $\ell_{0}$ norm $|\hat{\boldsymbol{a}}(t)|_{0}-\left|\boldsymbol{a}^{*}\right|_{0}$ is 10 , which is close to the value when the system is stable, the algorithm still gives priority to reducing the energy functions (i.e., the period before $|\hat{\boldsymbol{a}}(t)|_{0}-\left|\boldsymbol{a}^{*}\right|_{0}$ grows up to 140) in order to choose appropriate active nodes, and this procedure is finished in a split second.

Furthermore, one hundred Monte Carlo experiments are did to estimate $T_{M A X}$ with different $\beta$. Suppose the initial condition is generated from a Gaussian distribution with standard derivation $\sigma=5, \alpha=0.5$ is fixed, then we vary $\beta=[1.05,1.38,2,5,8,13,20]$. Besides, we present three results of the 100 , and each $T_{\max }$ is the largest converge time of $T_{\text {converge }}$ which corresponds to different $\beta$. Especially, $1.38<\beta=1+\frac{2}{\ln N}<2$ when $N=200$. The relationship between $T_{\max }$ and $\beta$ in Fig. 6 demonstrates that the minimum value of $T_{\max }$ occurs near $\beta=2$, which is a acceptable result.

At last, we present the convergence trajectory of four nodes under different initial conditions, respectively. Without loss of generality, we select nodes $\boldsymbol{x}_{10}, \boldsymbol{x}_{63}, \boldsymbol{x}_{108}$, and 


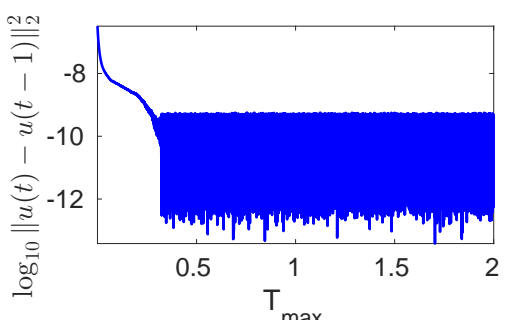

(a)

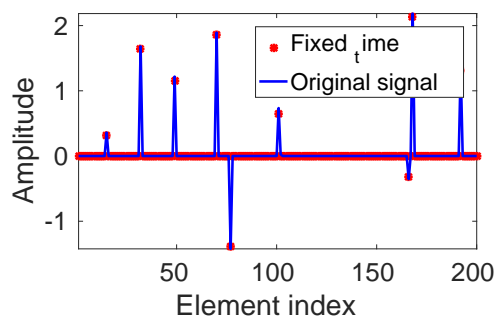

(c)

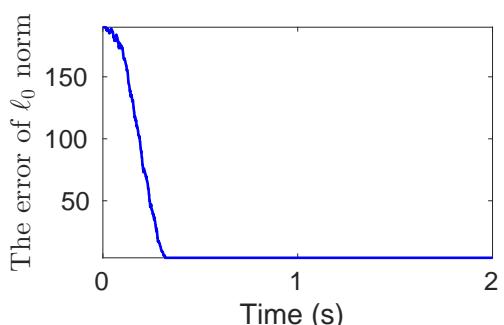

(b)

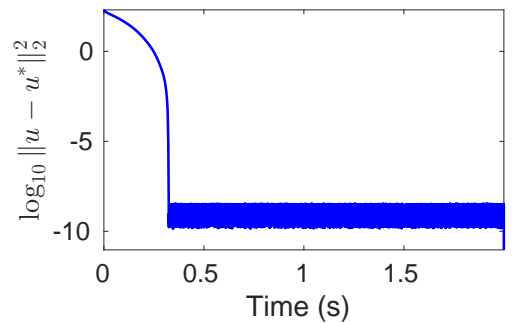

(d)

Figure 4: Convergence process with initial condition of $[1, \ldots 1]$.
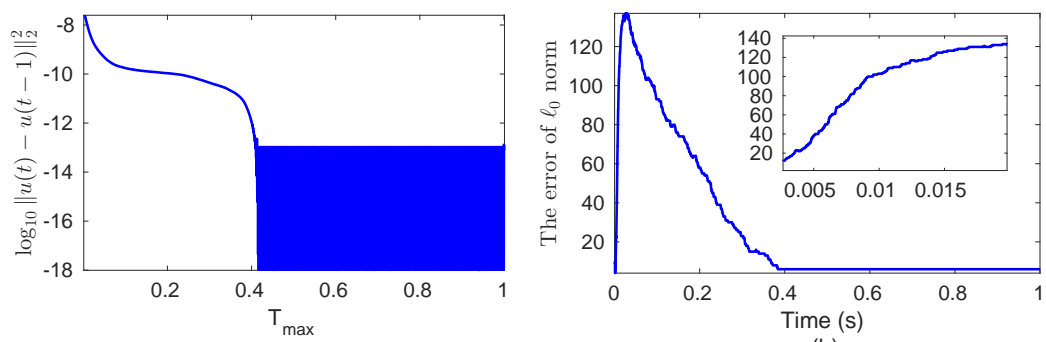

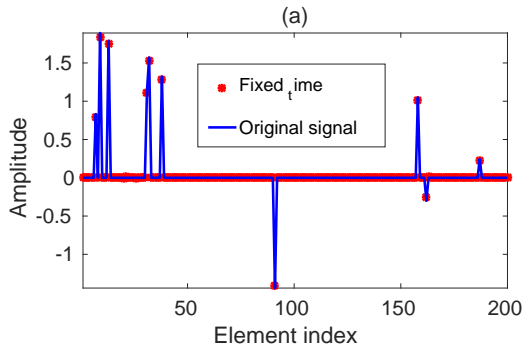

(c)

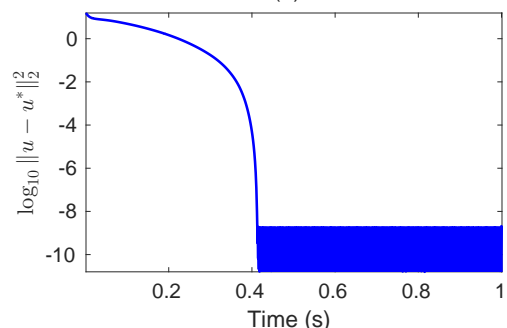

(d)

Figure 5: Convergence process with initial condition of $[0, \ldots 0]$. 

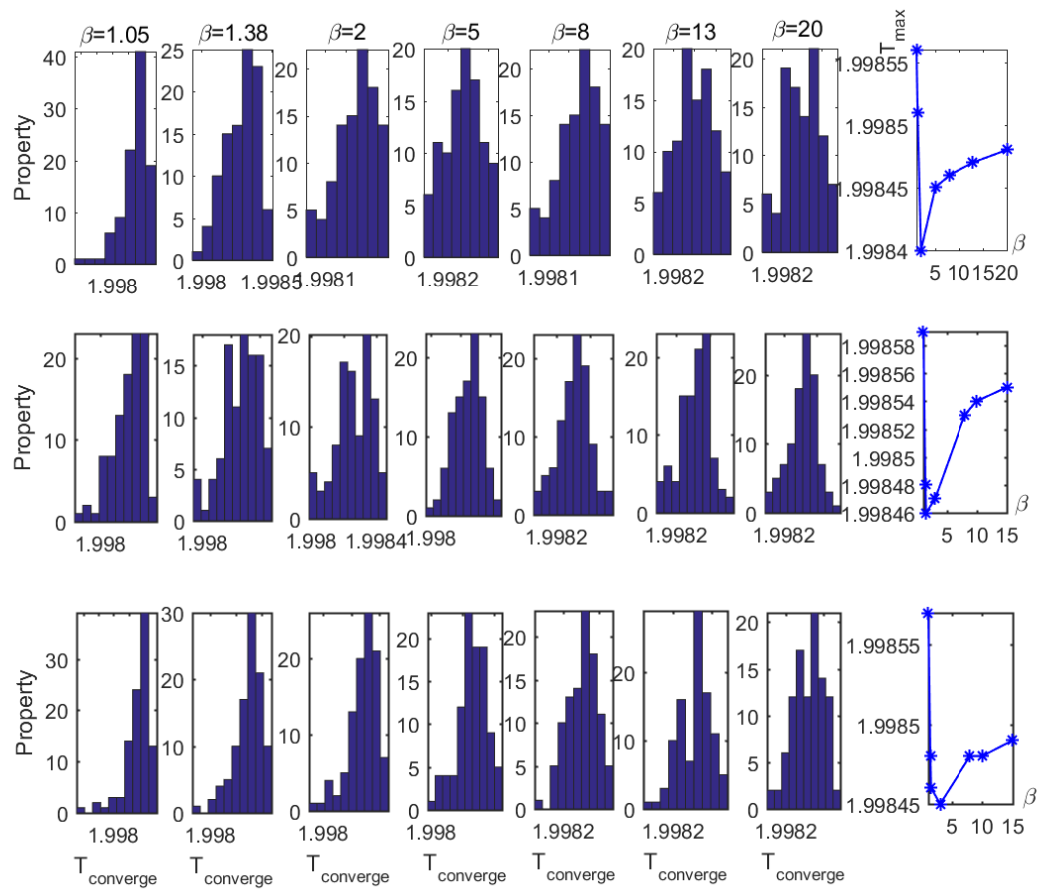

Figure 6: The hist of convergence time $T_{\text {converge }}$ under different $\beta$ and the relationship between $T_{\max }$ (the biggest $T_{\text {converge }}$ of each hist) and $\beta$. 
$x_{174}$ of the sparse signal $x$, then set $\beta=1.5, \alpha=0.2$. The simulation results in Fig. $\square$ show that each node stabilizes within $0.3 s$, which reflect the fixed-time convergence of the proposed system to some extent.
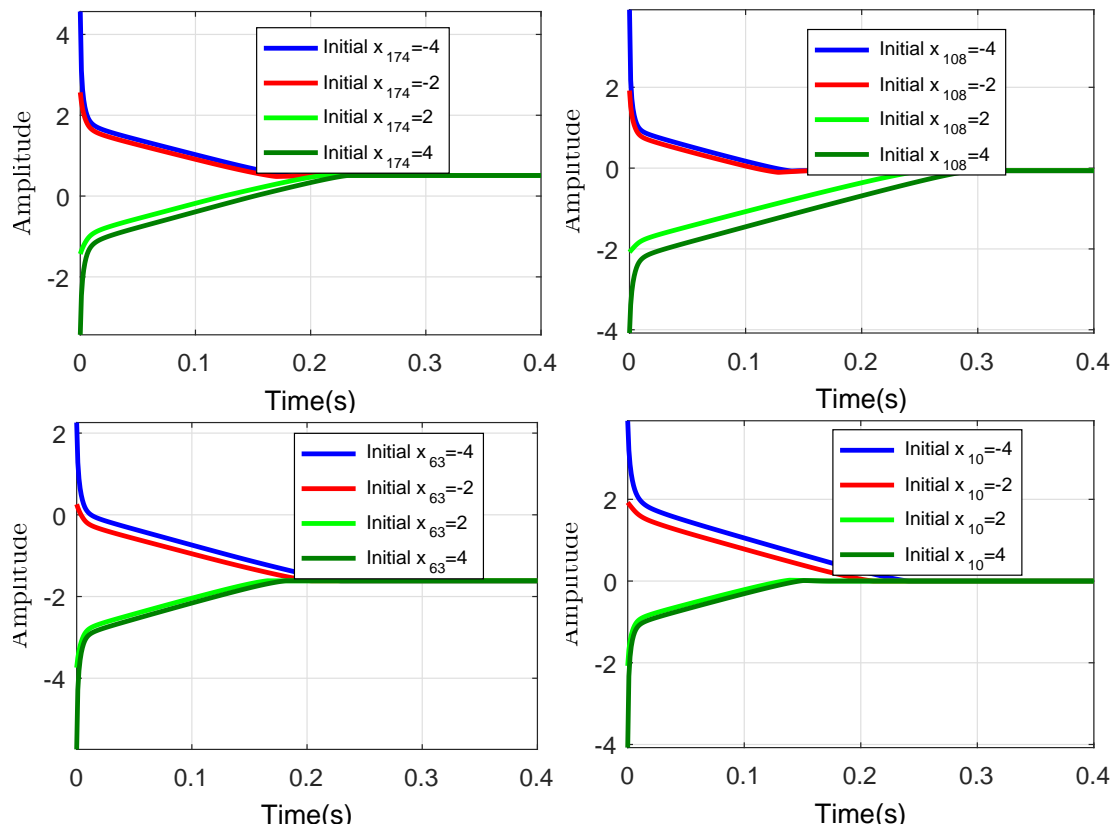

Figure 7: Convergence for different initial conditions of nodes number 174, 108, 63, and 10.

\subsection{Influence of $\alpha$ and $\beta$}

In this section, we mainly illustrate the influence of $\alpha$ and $\beta$ on convergence performance. Let us firstly consider the influence of $\alpha$ on the convergence rate. Fix $\beta=1.5$ and vary $\alpha$ in interval $[0.2,0.8]$ with step of 0.2 . All the other parameters will be maintained in their defaults. With comparison to LCA and finite-time system, the result in Fig. 8 reflects that the proposed system is faster than them with respect to the same $\alpha$. Moreover, the convergence rate is slower as $\alpha$ increases, which is consistent with the theoretical result in Remark 2 .

Then, we test the influence of $\beta$ on the convergence rate. Here we assume that $\alpha=0$ corresponds to non $\alpha$ term and not the usual sliding mode with $\alpha=0$, so is the $\beta$. Set the initial condition that all the nodes are 10. Observing the result in Fig. $Q$ with 


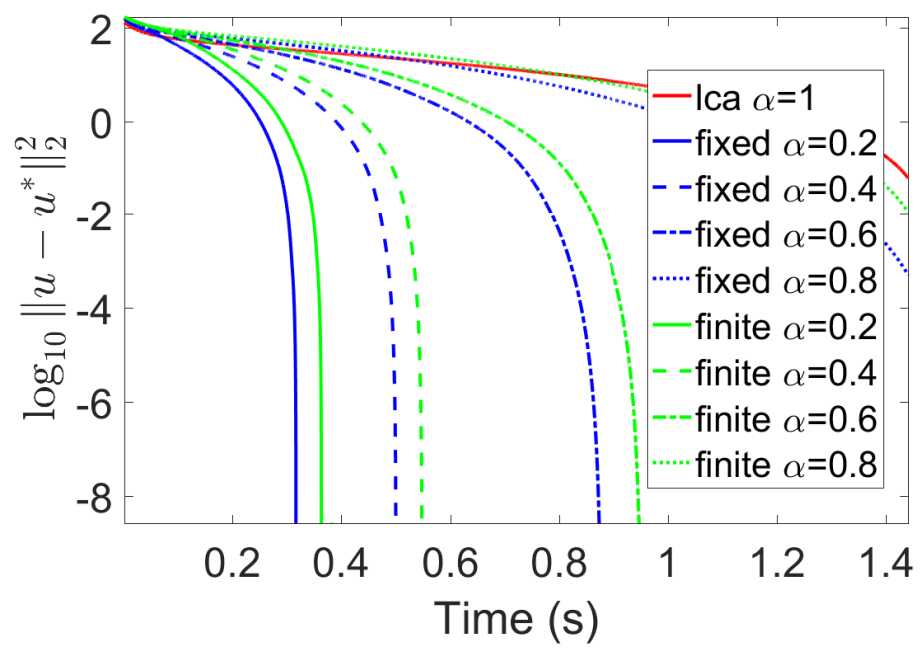

Figure 8: Convergence of $\left\|u-u^{*}\right\|_{2}^{2}$ for the LCA (solid red line), proposed dynamical system (solid lines), and finite-time system (dashed lines) according to different values of $\alpha$.

the same $\alpha=0$ but different $\beta$ ( $\beta=1.5$ or $\beta=3$ ), it reflects that without $\alpha$ term, the $\beta$ can ensure rapid convergence to the system while $\boldsymbol{u}(t)$ is far away from the equilibrium point. However, after $\boldsymbol{u}(t)$ reaches a certain distance to the equilibrium point, the system seems to be much slower and more difficult to approach the equilibrium point, which could explain the previous conclusion in Fig. 6 as well that the bigger $\beta$ is, the larger $T_{\max }$ is. After adding $\alpha$ term, the system can quickly converge when it approaches the equilibrium point. For example, it's obvious that the convergence rate of the system is much faster when $\alpha=0.2, \beta=3$ rather than $\alpha=0, \beta=3$. Meanwhile, the fixed-time system is always faster than finite-time system and LCA $(\alpha=1, \beta=0)$ with the common effects of both $\alpha$ and $\beta$.

\section{Conclusion}

In this paper, we proposed a fixed-time dynamical system to solve the general SR problem. The most important advantage of the proposed method is its fast convergence speed, meanwhile, $\alpha$ and $\beta$ will play an important role respectively when the system is near to or far away from the equilibrium point. In addition, the upper bound $T_{\max }$ of 


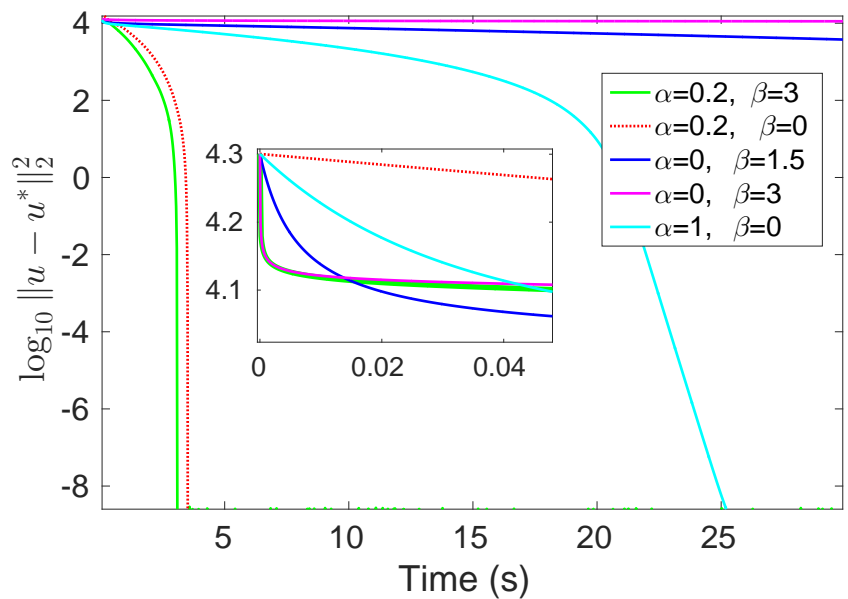

Figure 9: Convergence of $\left\|u-u^{*}\right\|_{2}^{2}$ for the proposed dynamical system with different setting of $\alpha$ and $\beta$.

convergence time is independent of the initial condition, and it is smaller with smaller $\alpha$. For $\beta$, it's better to choose $\beta \in(1,2)$ for $N>e^{2}$, with $e$ the irrational number $2.71828 \ldots$

\section{References}

[1] S. Qaisar, R. M. Bilal, W. Iqbal, M. Naureen, S. Lee, Compressive sensing: From theory to applications, a survey, Journal of Communications and Networks 15 (5) (2013) 443-456.

[2] S. Theodoridis, Y. Kopsinis, K. Slavakis, Sparsity-aware learning and compressed sensing: An overview, arXiv preprint arXiv:1211.5231.

[3] G. Kutyniok, Theory and applications of compressed sensing, GAMMMitteilungen 36 (1) (2013) 79-101.

[4] M. A. Davenport, M. F. Duarte, Y. C. Eldar, G. Kutyniok, Introduction to compressed sensing, Preprint 93 (1) (2011) 2.

[5] K. Hayashi, M. Nagahara, T. Tanaka, A user's guide to compressed sensing for 
communications systems, IEICE Transactions on Communications 96 (3) (2013) $685-712$.

[6] L. Yu, J. P. Barbot, G. Zheng, H. Sun, Bayesian compressive sensing for cluster structured sparse signals, Signal Processing 92 (1) (2012) 259-269.

[7] L. Yu, H. Sun, G. Zheng, J. P. Barbot, Model based bayesian compressive sensing via local beta process, Signal Processing 108 (3) (2015) 259-271.

[8] D. L. Donoho, Compressed sensing, IEEE Transactions on Information Theory 52 (4) (2006) 1289-1306.

[9] M. Elad, M. A. Figueiredo, Y. Ma, On the role of sparse and redundant representations in image processing, Proceedings of the IEEE 98 (6) (2010) 972-982.

[10] Z. Zhang, Y. Xu, J. Yang, X. Li, D. Zhang, A survey of sparse representation: algorithms and applications, IEEE Access 3 (2015) 490-530.

[11] E. J. Candes, T. Tao, Decoding by linear programming, IEEE Transactions on Information Theory 51 (12) (2005) 4203-4215.

[12] I. Daubechies, M. Defrise, C. De Mol, An iterative thresholding algorithm for linear inverse problems with a sparsity constraint, Communications on Pure and Applied Mathematics 57 (11) (2004) 1413-1457.

[13] S. S. Chen, D. L. Donoho, M. A. Saunders, Atomic decomposition by basis pursuit, SIAM Review 43 (1) (2001) 129-159.

[14] R. Tibshirani, Regression shrinkage and selection via the lasso, Journal of the Royal Statistical Society. Series B (Methodological) (1996) 267-288.

[15] R. Baraniuk, P. Steeghs, Compressive radar imaging, in 2007 IEEE Radar Conference. IEEE, apr (2007) 128-133.

[16] J. Wright, A. Y. Yang, A. Ganesh, S. S. Sastry, Y. Ma, Robust face recognition via sparse representation, IEEE Transactions on Pattern Analysis and Machine Intelligence 31 (2) (2009) 210-227. 
[17] X. Xu, X. Wei, Z. Ye, DOA estimation based on sparse signal recovery utilizing weighted $l_{1}$-norm penalty, IEEE Signal Processing Letters 19 (3) (2012) 155-158.

[18] C. J. Rozell, D. H. Johnson, R. G. Baraniuk, B. A. Olshausen, Sparse coding via thresholding and local competition in neural circuits, Neural Computation 20 (10) (2008) 2526-2563.

[19] A. Balavoine, J. Romberg, C. Rozell, Convergence and rate analysis of neural networks for sparse approximation, IEEE Transactions on Neural Networks and Learning Systems 23 (9) (2012) 1377-1389.

[20] A. Balavoine, C. J. Rozell, J. Romberg, Discrete and continuous-time softthresholding for dynamic signal recovery, IEEE Transactions on Signal Processing 63 (12) (2015) 3165-3176.

[21] A. Balavoine, J. Romberg, C. J. Rozell, Correction to "Convergence and Rate Analysis of Neural Networks for Sparse Approximation", IEEE Transactions on Neural Networks and Learning Systems 25 (8) (2014) 1595-1596.

[22] L. Yu, G. Zheng, J. P. Barbot, Dynamical sparse recovery with finite-time convergence, IEEE Transactions on Signal Processing 65 (23) (2017) 6146-6157.

[23] S. P. Bhat, D. S. Bernstein, Finite-time stability of continuous autonomous systems, SIAM Journal on Control and Optimization 38 (3) (2000) 751-766.

[24] Y. Orlov, Finite time stability and robust control synthesis of uncertain switched systems, SIAM Journal on Control and Optimization 43 (4) (2004) 1253-1271.

[25] A. Polyakov, Nonlinear feedback design for fixed-time stabilization of linear control systems, IEEE Transactions on Automatic Control 57 (8) (2012) 2106-2110.

[26] W. Lu, X. Liu, T. Chen, A note on finite-time and fixed-time stability, Neural Networks 81 (2016) 11-15.

[27] Z. Zuo, L. Tie, A new class of finite-time nonlinear consensus protocols for multiagent systems, International Journal of Control 87 (2) (2014) 363-370. 
[28] J.-J. Fuchs, On sparse representations in arbitrary redundant bases, IEEE Transactions on Information Theory 50 (6) (2004) 1341-1344.

[29] H. K. Khalil, Noninear systems, Prentice-Hall, New Jersey 2 (5) (1996) 5-1.

[30] Z. Zuo, L. Tie, Distributed robust finite-time nonlinear consensus protocols for multi-agent systems, International Journal of Systems Science 47 (6) (2016) $1366-1375$. 